\title{
ULTRASONIC ATTENUATION DUE TO THE NEUTRAL ACCEPTOR INDIUM IN SILICON
}

\author{
Hp. SCHAD and K. LASSMANN \\ Physikalisches Institut der Universität Stuttgart, Germany
}

Received 10 February 1976

The acceptor ground state $\Gamma_{8}$ of shallow acceptors in cubic semiconductors is partly split due to local fields even in clean crystals of low doping and dislocation density and the distribution of such level splittings may be rather wide [1].

For deep acceptors there is evidence that the ground state may be more complicated. In the case of $\mathrm{GaAs}(\mathrm{Mn})$ the analysis of our results [2] indicates that the ground state consists of a distribution of split levels (width $\sim 0.05 \mathrm{meV}$ ) and an excited level 3 meV above. Such a complication may be connected with a dynamic Jahn-Teller effect.

We have measured the low temperature and frequency dependencies of the u.s.-attenuation of a longitudinal u.s. wave in [111] excited by a thin film CdS-transducer in the wide frequency range 0.38 to $4 \mathrm{GHz}$.

Our sample was moderately doped with In (content $5 \times 10^{15} \mathrm{~cm}^{-3}$, dislocation density $\sim 10^{3} \mathrm{~cm}^{-2}$ ) and rather pure, as seen from luminescence measurements, apart from a contamination with oxygen (content $1.4 \times 10^{18} \mathrm{~cm}^{-3}$, obtained from the absorption in the near infrared). We have checked the influence of oxygen on the u.s. attenuation with a second sample, which was free of indium and doped with $1.8 \mathrm{X}$ $10^{18} \mathrm{~cm}^{-3}$ oxygen, and have found no effect between $300 \mathrm{~K}$ and $1 \mathrm{~K}$ at $1 \mathrm{GHz}$. Therefore, we are convinced that the results described below are due to the indium acceptor.

Fig. 1 shows the main features of our results. For high temperatures the attenuation is proportional to $T^{4}$ due to phonon-phonon interaction. For low temperatures and small acoustic intensities there is a rise $\propto 1 / T$. Such a behaviour is expected for resonance interaction between the u.s. wave and a distribution of level splittings $\delta$ of the acceptor ground state. We estimate from the absolute value of the attenuation a width of this distribution of approximately $0.1 \mathrm{meV}$.

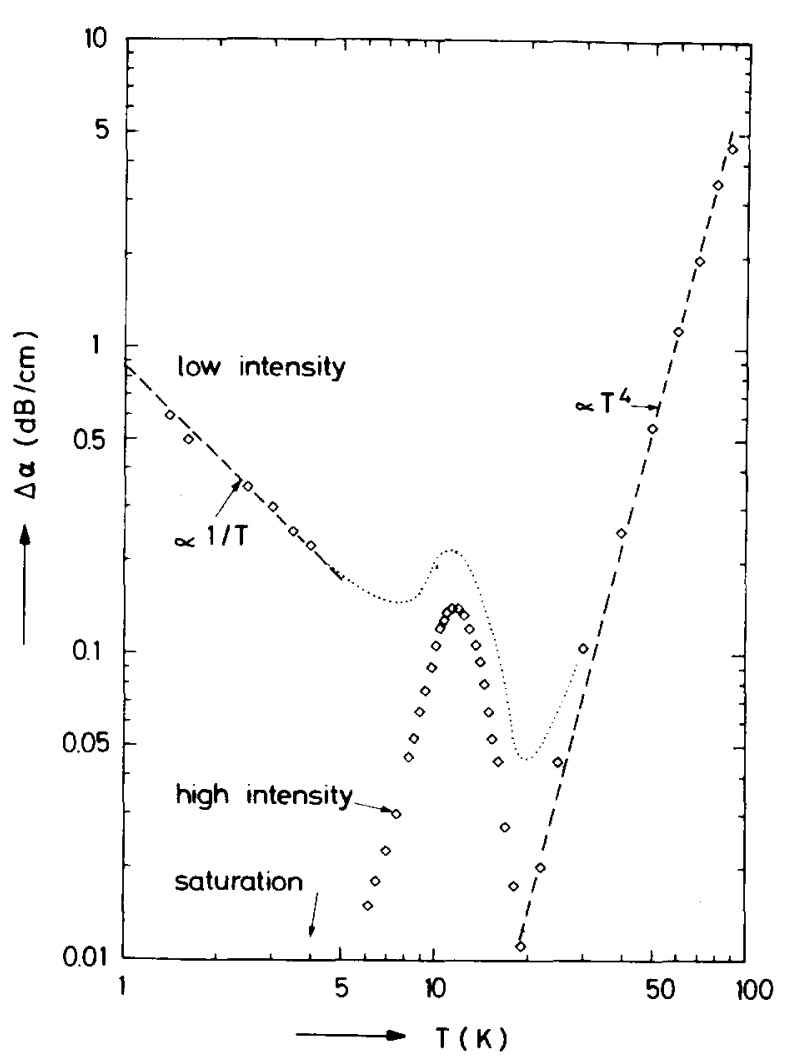

Fig. 1. Temperature dependence of the attenuation; $3 \mathrm{GHz}$.

For high acoustic intensities this rise vanishes following the relation $\alpha_{\text {res }} \propto I^{-1 / 2}(I=$ acoustic intensity), as was found in the u.s. attenuation in glasses [e.g. 3]. Such a saturation effect of the u.s. attenuation by the acceptor ground state has been seen here as well as in the case of shallow acceptors in $\mathrm{Ge}$ [1] for the first time and will be discussed in more detail elsewhere.

In the intermediate temperature range there is an attenuation peak, as was found similarly in $\mathrm{GaAs}(\mathrm{Mn})$ [2] and also in Si (B) [4]. The peak height is propor- 


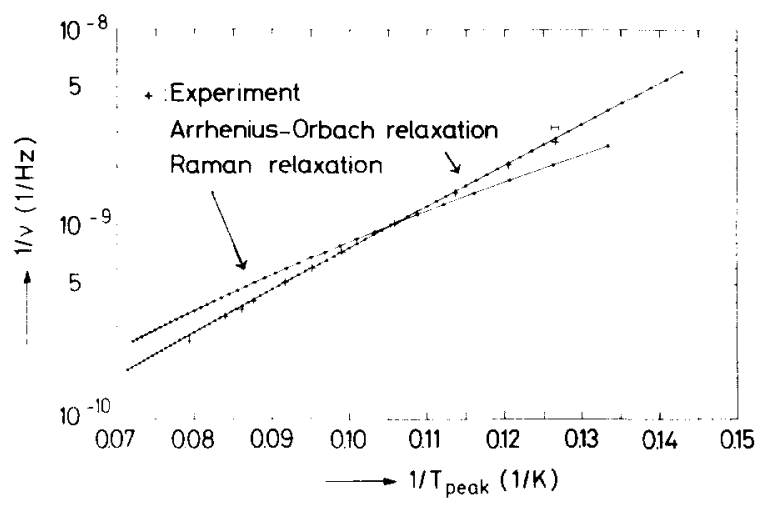

Fig. 2. Frequency dependence of the peak maximum temperature.

tional to frequency $\nu$ and its maximum temperature shifts with frequency to higher temperatures. We interpret this peak as due to relaxation. For $\delta \lesssim 0.1 \mathrm{meV}$ the relaxation formula is approximately given by [2]:

$\alpha_{\mathrm{rel}} \propto\left(D^{\prime 2} / T\right) \cdot \nu^{2} \tau(T) /\left(1+(2 \pi \nu)^{2} \tau^{2}(T)\right)$

where $D^{\prime}$ is an effective deformation potential constant and $\tau(T)$ the relaxation time of the corresponding relaxation process, assumed to be independent of $\delta$ (relaxation time $\tau(T, \delta)$ due to direct processes can be excluded). The relaxation time can be determined from the peak maximum (approximate condition $2 \pi \nu \tau$ $\left.\left(T_{\max }\right)=1\right)$. Measuring at high echo numbers, with the intensity still high enough to saturate and thus eliminate the additional resonance attenuation, we were able to determine the relative attenuation with high resolution by recording several echoes continuously with varying temperature. $T_{\max }$ was accurate to $\pm 0.05 \mathrm{~K}$ for the lower and $\pm 0.1 \mathrm{~K}$ for the higher temperatures. In our calculations (taking into account the $1 / T$ dependence of the prefactor in the relaxation formula) the best fit of the experimental frequency dependence of $T_{\max }$ is given by an Arrhenius equation $\tau_{\mathrm{A}}(T) \propto \exp \left(\Delta / k_{\mathrm{B}} T\right)$ for the relaxation time (fig. 2). This indicates an Orbach relaxation process with an excited level $\Delta=4.2 \pm 0.3 \mathrm{MeV}$ above the level splittings $\delta$.

In fig. 2 we have also plotted the frequency dependence of $T_{\max }$ to be expected for Raman relaxation with the relaxation time $\tau_{\mathrm{R}}^{-1} \propto D^{\prime \prime} T^{5} f(T)(f(T)=$ cutoff function, which describes the reduced interaction of the Raman phonons with shorter wavelength than the Bohr radius of $7.3 \AA$, and $D^{\prime \prime}=$ effective de- formation potential fitted in fig. 2 for coincidence with experiment at $1 \mathrm{GHz}$.) Clearly, the Raman relaxation does not describe the experimental results. Furthermore, taking for $D^{\prime \prime}$ the experimentally determined value of $\mathrm{Si}(\mathrm{In})$, the Raman peak would appear at higher temperatures and would be far too broad, whilst an Arrhenius peak fits rather well the observed attenuation. A combination of both relaxations ( $\mathrm{Ra}$ man relaxation with experimental deformation poten. tials) modifies only to a small amount the ArrheniusOrbach results.

In summary we find that for $\mathrm{Si}(\mathrm{In})$ there is a level $4.2 \mathrm{meV}$ above the ground state as similarly found for GaAs (Mn); it appears that such a level is characteristic for deep acceptors. Corresponding resonance energies have also been seen in the low temperature thermal conductivity measurements with the same samples [5].

In contrast to these results preliminary measurements in the far infrared and at very high phonon frequencies with superconducting tunnel junctions did not reveal a line at $4.2 \mathrm{meV}$ (which may be due to the low content of indium) but only the small absorption line of oxygen at $3.6 \mathrm{meV}[6,7]$.

We thank W. Arnold (MPIF, Grenoble) for the suply of transducers, J. Kuhl and his coworkers (MPIF, Stuttgart) for far and near infrared measurements, and the following colleagues of the Physikalisches Institut, Stuttgart: W. Forkel, R. Sauer and R. Grill for doing phonon spectroscopy, luminescence and far infrared measurements, respectively, and W. Eisenmenger for constant interest and many discussions.

\section{References}

[1] E. Ortlieb, Hp. Schad and K. Lassmann, Proc. 2nd Intern. Conf. on Phonon scattering in solids, to be published.

[2] K. Lassmann and Hp. Schad, Sol. State Comm., to be published.

[3] S. Hunklinger et al., Phys. Lett. 45A (1973) 311.

[4] T. Ishiguro and H. Tokumoto, J. Phys. Soc. Japan 37 (1974) 1716.

[5] A. de Combarieu and K. Lassmann, Proc. 2nd Intern. Conf. on Phonon scattering in solids, to be published.

[6] W. Hayes and D.R. Bosomworth, Phys. Rev. Lett. 23 (1969) 851 .

[7] W. Forkel, M. Welte and W. Eisenmenger, Phys. Rev. Lett. 31 (1973) 215. 